\title{
Continuous intracranial pressure monitoring in severe traumatic brain injury in children
}

\author{
St.M. Iencean ${ }^{1,2}$, A. Tascu ${ }^{3,4}$, C.A. Apetrei ${ }^{2}$, C. Gheorghita ${ }^{5}$, \\ Tsz-Yan Milly Lo ${ }^{6}$, Ian Piper ${ }^{7}$, A.St. Iencean ${ }^{2}$
}

${ }^{1}$ Neurosurgery, "Grigore T. Popa" University of Medicine and

Pharmacy, Iasi, ROMANIA

2 Neurosurgery, "Prof. Dr. N. Oblu" Clinical Emergency Hospital, Iasi, ROMANIA

${ }^{3}$ Neurosurgery, "Bagdasar-Arseni" Clinical Emergency Hospital, Bucharest, ROMANIA

4 Neurosurgery, "Carol Davila" University of Medicine and Pharmacy, Bucharest, ROMANIA

5 Neurosurgery, "Sf. Maria" Children Clinical Emergency Hospital, Iasi, ROMANIA

${ }^{6}$ University of Edinburgh (Child Life \& Health) / Royal Hospital for Sick Children (Paediatric Critical Care Medicine), UK

${ }^{7}$ Brain IT Group Coordinator, Principal Health Care Scientist, NeuroIntensive Care Monitoring Research, UK

\begin{abstract}
We present the results of the Romanian team for the multi-center grant "Paediatric Brain Monitoring with Information Technology (KidsBrainIT). Using IT Innovations to Improve Childhood Traumatic Brain Injury Intensive Care Management, Outcome, and Patient Safety", acronym KidsBrainlT. Children aged 2 to 16 years who require intensive care management after sustaining traumatic severe brain injury are included in this study in three neurosurgical hospital: "Prof. Dr. N. Oblu" Clinical Emergency Hospital Iasi, "Sf. Maria" Children Clinical Emergency Hospital lasi and "Bagdasar-Arseni" Clinical Emergency Hospital Bucharest. Continuous real-time intracranial pressure monitoring became a "gold standard" in TBI intensive-care management and ICP-lowering therapy is recommended when ICP is elevated above $20 \mathrm{mmHg}$ or more. Continuous ICP and mean arterial blood pressure (MAP) monitoring allow calculation of cerebral perfusion pressure (CPP) and to establish of an optimal CPP. This study aims to improve the treatments and the outcomes in severe traumatic brain injury in children.
\end{abstract}

\section{INTRODUCTION}

Annually, over 50,000 new cases of cranio-cerebral trauma (TBI) occur in Romania; road accidents are the main cause of cranial traumas, which often cause cognitive, affective and behavioural disorders, with a particular impact on families and society. World Health Organization
Keywords

cerebral perfusion pressure,

traumatic coma,

intracranial pressure,

paediatric brain monitoring,

severe children brain injury

$\triangle$

Corresponding author

A. Tascu

"Carol Davila" University of

Medicine and Pharmacy,

Bucharest, Romania

tascu_alexandru@yahoo.com

Copyright and usage. This is an Open Access article, distributed under the terms of the Creative Commons Attribution Non-Commercial No Derivatives License (https:/creativecommons org/licenses/by-nc-nd/4.0/) which permits noncommercial re-use, distribution, and reproduction in any medium, provided the original work is unaltered and is properly cited.

The written permission of the Romanian Society of Neurosurgery must be obtained for commercial re-use or in order to create a derivative work.

ISSN online 2344-4959

(C) Romanian Society of Neurosurgery

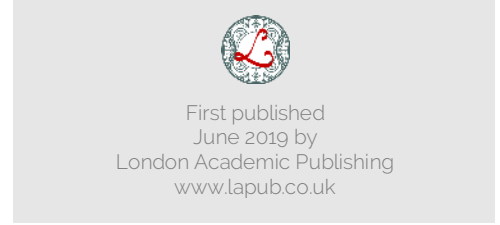


estimated that up to $90 \%$ of head injuries that receive treatment are mild, of which moderate and severe injuries represent $10 \%$. In the European Union the yearly aggregate incidence of TBI hospitalizations and fatalities is estimated at 235 per 100,000 .

Fortunately, TBI in children is much lower, without being able to make a reliable estimate because of research differences, but partial studies have shown that $\mathrm{TBI}$ in children represents about $14 \%$ of the total TBI. Also, children have a higher incidence of increased intracranial pressure (ICP) following TBI than adults ( $80 \%$ vs. $50 \%$ ) and it is a major cause of morbidity and mortality in the paediatric age group.

In this report of the multi-center grant "Paediatric Brain Monitoring with Information Technology (KidsBrainIT). Using IT Innovations to Improve Childhood Traumatic Brain Injury Intensive Care Management, Outcome, and Patient Safety", acronym KidsBrainIT we present the results of our study for almost the past two years.

\section{MATERIAL AND METHODS}

Three neurosurgical hospital: "Prof. Dr. N. Oblu" Clinical Emergency Hospital Iasi, "Sf. Maria" Children Clinical Emergency Hospital lasi and "BagdasarArseni" Clinical Emergency Hospital Bucharest participated in this study that included children aged 2 to 16 years who require intensive care management after sustaining traumatic severe brain injury. A total of 941 children with traumatic brain injury received medical care during 16 months in these three neurosurgical departments, including minor, medium and severe brain traumas. Thirtyone patients needed intensive care and 9 children have been ICP and blood pressure monitored, but only four patients were included in this scientific project. As presented in the previous report in two cases the values of ICP were high and very high and cerebral decompression was performed; unfortunately, the initial clinical condition was extremely severe and evolution was not favourable in these cases. The third and fourth patients monitored showed elevated ICP values up to 28-30 $\mathrm{mm} \mathrm{Hg}$ and $30-40 \mathrm{~mm} \mathrm{Hg}$, which were medically treated.

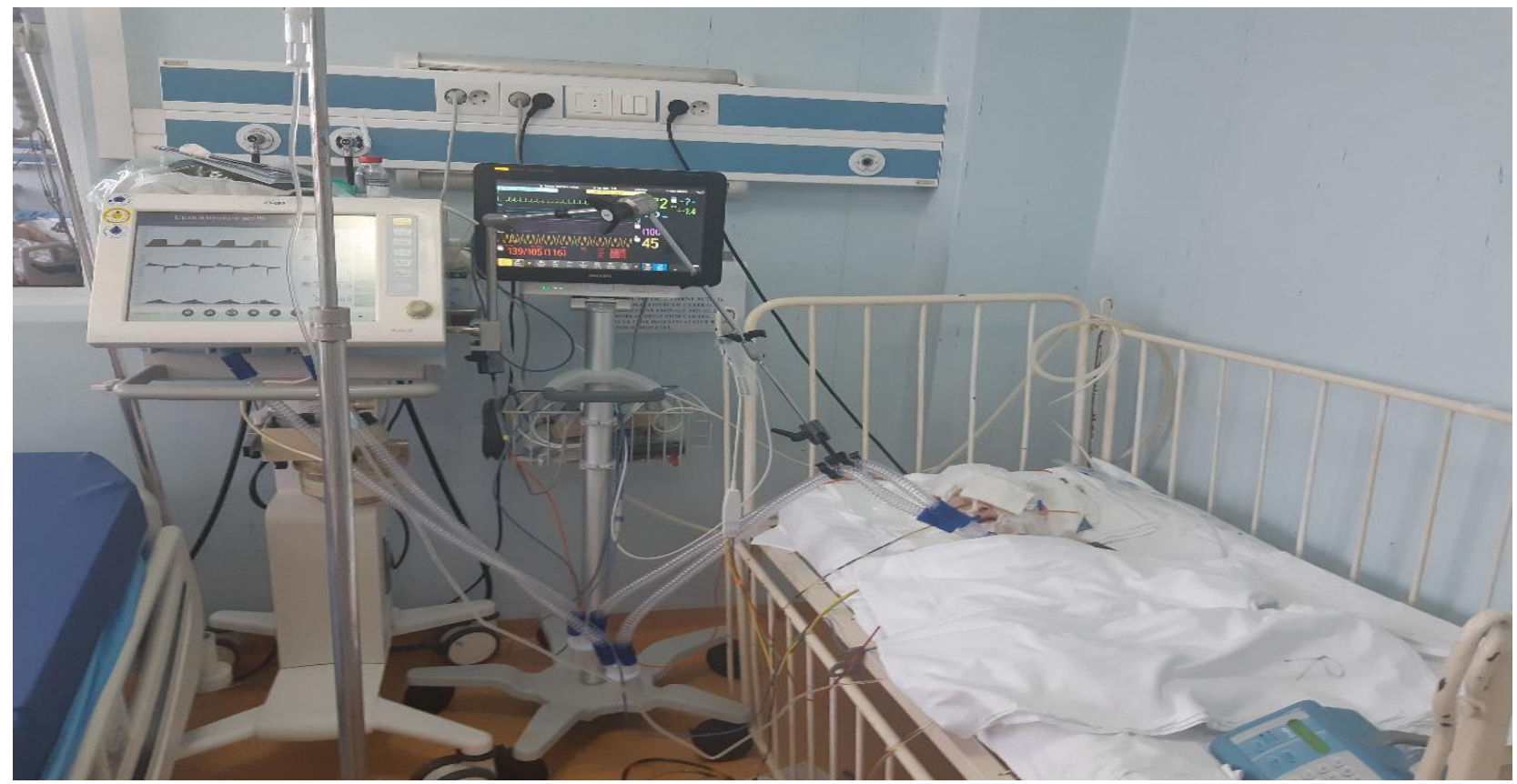

FIGURE 1: Case of children with severe TBI and continuous intracranial pressure monitoring

\section{RESULTS}

During 16 months in our three neurosurgical departments there were a total of 941 children with traumatic brain injury and 31 patients needed intensive care and 9 children have been ICP and blood pressure monitored, but only four patients were included in this scientific project. The scientific report that on the mid-term results of this multicentre grant presented the three cases: two children 
with high and very high values of ICP and cerebral decompression, but not favourable evolution and the case of the third patient monitored with elevated ICP values up to $28-30 \mathrm{~mm} \mathrm{Hg}$, medically treated with a favourable evolution. The four case had oscillating high ICP values up to $30-45 \mathrm{~mm} \mathrm{Hg}$, but the extremely severe initial clinical condition has made its evolution not favourable.

\section{DISCUSSION}

Our number of 941 cases in 16 months should be interpreted by reference only for two areas in the country: two hospitals in lasi and one in Bucharest, so it does not cover the entire our country. This number of cases of paediatric TBI vary across clinical and epidemiological studies and till now our number of cases with ICP and CPP monitoring are few and a statistical analysis could not be conclusive, but all the cases from the centres included in the grant from the other countries were hoping to have a statistical significance.

Intracranial pressure and cerebral perfusion pressure monitoring are invasive methods but they assured an early detection of increased ICP and disturbance of cerebral perfusion pressure in children with severe TBI. As we have mentioned in another material about this grant, the relationship between ICP elevation and CPP values is known in the adult, but in the paediatric TBI the studies are not conclusive; so "Chambers et al. proposed age stratified critical levels of CPP: in the age groups 2-6, 7-10, and 11-16 years, CPP values of $43 \mathrm{mmHg}, 54$ $\mathrm{mmHg}$ and $58 \mathrm{mmHg}$, respectively, were associated with normal values of ICP and good outcomes".

Within the partnership between the centres involved in the finalization of this scientific research it has been achieved a distribution of the research work packages/tasks by partner: (1) Kids BrainIT infrastructure development; (2) multi-centre multinational patient recruitment into the project; (3) data extraction, central data-bank development and implementation; (4) outcome assessment (6 and 12 months); (5) data artefact detection and cleaning; (6) physiological data analyse; (7) novel technology substudy and (8) MRI outcome sub-study.

Anonymised clinical and physiological data from different centres are uploaded successfully into the data-bank using a secure http data upload service which ensures secured and encrypted transfer of fully anonymised data only. There are 48 patients recruited successfully into the study to-date in all contributing centres.

An important novel technology sub-study refers to the hybrid diffuse optical technology device, which is available for testing in Barcelona and patients are recruited into this sub-study since Barcelona open for recruitment in May 2018.

In our recruited patients with ICP and CPP monitoring the performed manoeuvres were drug therapy, CSF drainage and decompressive craniectomy in accordance with modern therapeutic guidelines. Treatment used sedatives, analgesics; hyperosmolar therapy as intravenous mannitol and hypertonic saline to control intracranial hypertension; mild hyperventilation; barbiturates, temperature control and prophylactic anticonvulsants. The routine steroid treatment in children with severe TBI is not conclusive; it has to be individualized and rather it is not indicated because the potential harm from infectious complications. Decompressive craniectomy was performed for controlling intracranial hypertension and it was effective at ICP reduction.

\section{CONCLUSIONS}

The severe traumatic brain injury in children requires a multidisciplinary approach in each phase of management. The prompt diagnosis during the initial evaluation and then the multimodal monitoring must be followed by the management of intracranial hypertension. Continuous ICP and mean arterial blood pressure (MAP) monitoring allow calculation of cerebral perfusion pressure (CPP) and to establish of an optimal CPP. All of this has its role to minimize the pathophysiological damage to the brain. The goal of this study is to establish the best care for severe TBI children and to ensure a transfer to a wider clinical audience.

\section{ACKNOWLEDGMENTS}

This study is within the grant: "Paediatric Brain Monitoring with Information Technology

(KIdsBrainIT): Using IT Innovations to Improve Childhood Traumatic Brain Injury Intensive Care Management, Outcome, and Patient Safety", grant: COFUND-NEURON III ERANET KidBrainIT, funding no.2 / 01/06/2017.

\section{REFERENCES}

1. Tsz-Yan Milly Lo. Paediatric Brain Monitoring with Information Technology (KIdsBrainIT): Using IT Innovations to Improve Childhood Traumatic Brain Injury Intensive 
Care Management, Outcome, and Patient Safety. Proposal Application Form - ERA-NET NEURON, 2016.

2. lencean St M, Tascu A, Apetrei CA, Gheorghita C,lencean A St. Continuous intracranial pressure monitoring in severe traumatic brain injury in children. Romanian Neurosurgery, Vol XXXII, Sept 2018, Supplement pp.73.

3. Kannan, N., Ramaiah, R., \& Vavilala, M. S. (2014). Pediatric neurotrauma. International journal of critical illness and injury science, 4(2), 131-137. DOI: 10.4103/2229-5151. 134152.

4. C.A. Apetrei, C. Gheorghita, A. Tascu, A.St. lencean, Tsz-Yan Milly Lo, Ian Pipe, St.M. lencean Paediatric Brain Monitoring with Information Technology (KidsBrainIT) - ERA-NET NEURON Grant. Romanian Neurosurgery (2018) XXXII 2: 183 186 DOI: 10.2478/romneu-2018-0024.

5. St.M. lencean, A. Tascu, C.A. Apetrei, C. Gheorghita, Tsz-Yan Milly Lo, Ian Piper, A.St. Iencean Mid-term results in continuous intracranial pressure monitoring in severe traumatic brain injury in children - ERA-NET NEURON Grant Romanian Neurosurgery (2018) XXXII 4: 547 - 551 DOI: 10.2478/romneu-2018-0070.

6. Tsz-Yan Milly Lo. Paediatric Brain Monitoring with Information Technology (KIdsBrainIT): Using IT Innovations to Improve Childhood Traumatic Brain Injury Intensive Care Management, Outcome, and Patient Safety. Annual Scientific Progress Report ERA-NET NEURON, April 2019.

7. Guidelines for the acute medical management of severe traumatic brain injury in infants, children, and adolescents (Second Edition). Pediatr Crit Care Med 2012. 13, No 1 (Suppl.).

8. Chambers IR, Jones PA, Lo TYM et al. Critical thresholds of intracranial pressure and cerebral perfusion pressure related to age in pediatric head injury. I Neurol Neurosurg Psychiatry 2006. 77(2): 234-240.

9. Depreitere B, Güiza F, Van den Berghe G, Schuhmann M, Maier G, Piper I, Meyfroidt G. Pressure autoregulation monitoring and cerebral perfusion pressure target recommendation in severe traumatic brain injury patients based on minute-by-minute monitoring data. J. Neurosurgery 2014 Jun; 120(6): 1451-1457.

10. Güiza F, Meyfroidt G, Lo TYM, Jones PA, Greet Van den B, Depreitere B. Continuous optimal CPP based on minuteby-minute monitoring data: a study on a pediatric population. Acta Neurochir 2015.

11. Guiza F, Depreitere B, Piper I et al. Visualizing the pressure and time burden of intracranial hypertension in adult and paediatric traumatic brain injury. Intensive Care Medicine 2015. 41(6): 1067-1076.

12. Hutchison JS, Frndova $H$, Lo TYM et al. Impact of hypotension and low cerebral perfusion pressure on outcomes in children treated with hypothermia therapy following severe traumatic brain injury: a post hoc analysis of the Hypothermia Pediatric Head Injury Trial. Dev Neurosci. 2010; 32(5-6): 406-12.

13. Suttipongkaset $P$, Chaikittisilpa $N$, Vavilala MS, Lele $A V$, Watanitanon A, Chandee T, Krishnamoorthy V. Blood Pressure Thresholds and Mortality in Pediatric Traumatic Brain Injury. Pediatrics. 2018;142(2).

14. Centers for Disease Control and Prevention. Report to congress: the management of traumatic brain injury in children. National Center for Injury Prevention and Control; Division of Unintentional Injury Prevention, Atlanta, GA; 2018.

15. Olsen, Mari et al. Incidence and mortality of moderate and severe traumatic brain injury in children: A ten-year population-based cohort study in Norway European Journal of Paediatric Neurology, Online May 2019. In Press.

16. Manfiotto $M$ et al. Decompressive craniectomy in children with severe traumatic brain injury: a multicentre retrospective study and literature review. World Neurosurgery. Available online 1 May 2019, In Press. 